\title{
Review of: "Determination of probability of causative pathogen in infectious keratitis using deep learning algorithm of slit-lamp images"
}

\author{
Merle Fernandes ${ }^{1}$ \\ 1 L V Prasad Eye Institute
}

Potential competing interests: The author(s) declared that no potential competing interests exist.

This is an interesting and well written manuscript on the application of deep learning algorithm for microbial keratitis images.

The authors have clubbed the different etiologies into 4 major categories : bacterial, fungal, acanthamoeba and viral. Organisms like Nocardia, atypical mycobacteria, phythium insidosum and microsporidia have typical clinical features which are probably not captured in this analysis.

To strengthen the possibility of making an accurate diagnosis, have the authors considered including salient points from history (ex trauma with vegetative matter may be associated with fungal keratitis), duration (ex longer duration may be associated with fungal keratitis while shorter duration may be associated with bacterial keratitis), and recurrent episodes (may be associated with HSV)?

Were the false positives and false negatives analyzed further to understand why there was an error?

There were a higher number HSV cases mis-diagnosed as bacteria. Did the authors notice whether fluorescein images had an additional benefit in diagnosing HSV keratitis? 